\title{
Design of control strategies to improve grid integration in fixed speed wind energy systems with battery storage
}

\author{
A. Goikoetxea ${ }^{1}$, M. Rodríguez ${ }^{1}$, H. Bindner ${ }^{2}$, A. Milo $^{3}$ \\ ${ }^{1}$ Faculty of Engineering, University of Mondragon, Loramendi 4, 20500 Mondragon, Spain, e-mail: \\ agoikoetxea@eps.mondragon.edu, marodriguez@eps.mondragon.edu \\ ${ }^{2}$ Risø National Laboratory, Technical University of Denmark - DTU, 4000 Roskilde, Denmark, e-mail: \\ henrik.bindner@risoe.dk
}

${ }^{3}$ Department of Control Engineering, IKERLAN, Research centre, Mondragon- Po J.M. Arizmendiarrieta, 2 (Spain), e-mail: amilo@ikerlan.es

\begin{abstract}
:
This article presents a study scenario composed of a fixed speed wind turbine and a DStatcom with battery storage, connected to the distribution grid as an isolated system.

The aim of the study is to demonstrate by means of simulating results how the DStatcom with storage can be a solution for a larger and better integration of the intermittent energy produced by DG in the electrical grid.

In this paper will be developed models and control strategies for the DStatcom with storage to make the complete system behave as a conventional power plant, providing voltage and frequency support (primary control) during normal operation and fault operation (voltage dips).
\end{abstract}

\section{Key words:}

Distributed Generation, Fixed Speed Wind Turbine, DStatcom, Battery Storage, grid integration control strategies.

\section{Introduction}

The electrical grid is one of the largest infrastructures that human has built. Up to few years ago, the power was produced in large generation centres and transported in high voltage (HV) to the consumption points, changing then to medium voltage (MV) for its distribution and to low voltage (LV) for its consumption. Due to environmental issues (climate changing, nuclear wastes...) and high price of fossil fuels renewable energy sources have become an alternative to reduce the consumption of traditional energy sources for electricity generation. The development of renewable energies in Spain has been mainly based on large wind farms connected to the HV transport grid and in a much lesser extent on small solar power plants.

For the following years is expected an important growth in renewable energy sources connected to the MV distribution grid. This increasing penetration of distributed generation (DG) devices is changing the topology of the electrical grid [1]. Even though the impact of these DG generators on the power quality is negligible nowadays, it will become very important in few years.
In this context of large penetration of DG, is interesting to analyse how a DStatcom (Distribution Static Compensator) with storage can help for a larger integration of the power produced by DG [2]. Shaving the peaks of DG power generation, storing this surplus power in a battery, and returning this energy during the production valleys the power connected to the grid is less variable. In this way is possible to reduce the impact on the voltage levels of the grid and the perturbations on the grid protection devices.

In this context the aims of this paper are to present a simulation scenario and develop the control strategies for the DStatcom with storage.

Next sections will describe the scenario proposed for the study, models of the devices and the control strategies proposed.

\section{Scenario for the study}

One of the most promising technologies for improving the integration of DG in the electrical grid is the Statcom with energy storage (STATCOM-ES) applied to fixed speed wind farms [2]. With this application is possible to control the active and reactive power generation for a fixed speed wind turbine. The proposed scenario is a low scale platform developed in the Syslab, this is a test facility at Risø National Laboratory very suitable to compare experimental and simulation results, and implement the control strategies developed in this paper $[3,4]$.

This facility is the perfect environment to analyse control strategies for the integration of DG. The microgrid is composed of $60 \mathrm{kVA}$ diesel generator set, $11 \mathrm{~kW}$ Gaia wind turbine, $55 \mathrm{~kW}$ Bonus wind turbine, $75 \mathrm{~kW}$ dump load, 45kVA back-to-back power converter, $10 \mathrm{~kW}$ deferrable load and 15kW/120kWh Vanadium battery.

For the present study the scenario proposed is composed of a $55 \mathrm{~kW}$ wind turbine connected to low voltage $400 \mathrm{v}$ three-phase grid, supported by a Redox battery of $120 \mathrm{kWh}$ [5] connected to the grid by means of a $15 \mathrm{Kw}$ inverter.

The topology of this system is presented in figure 1 . 


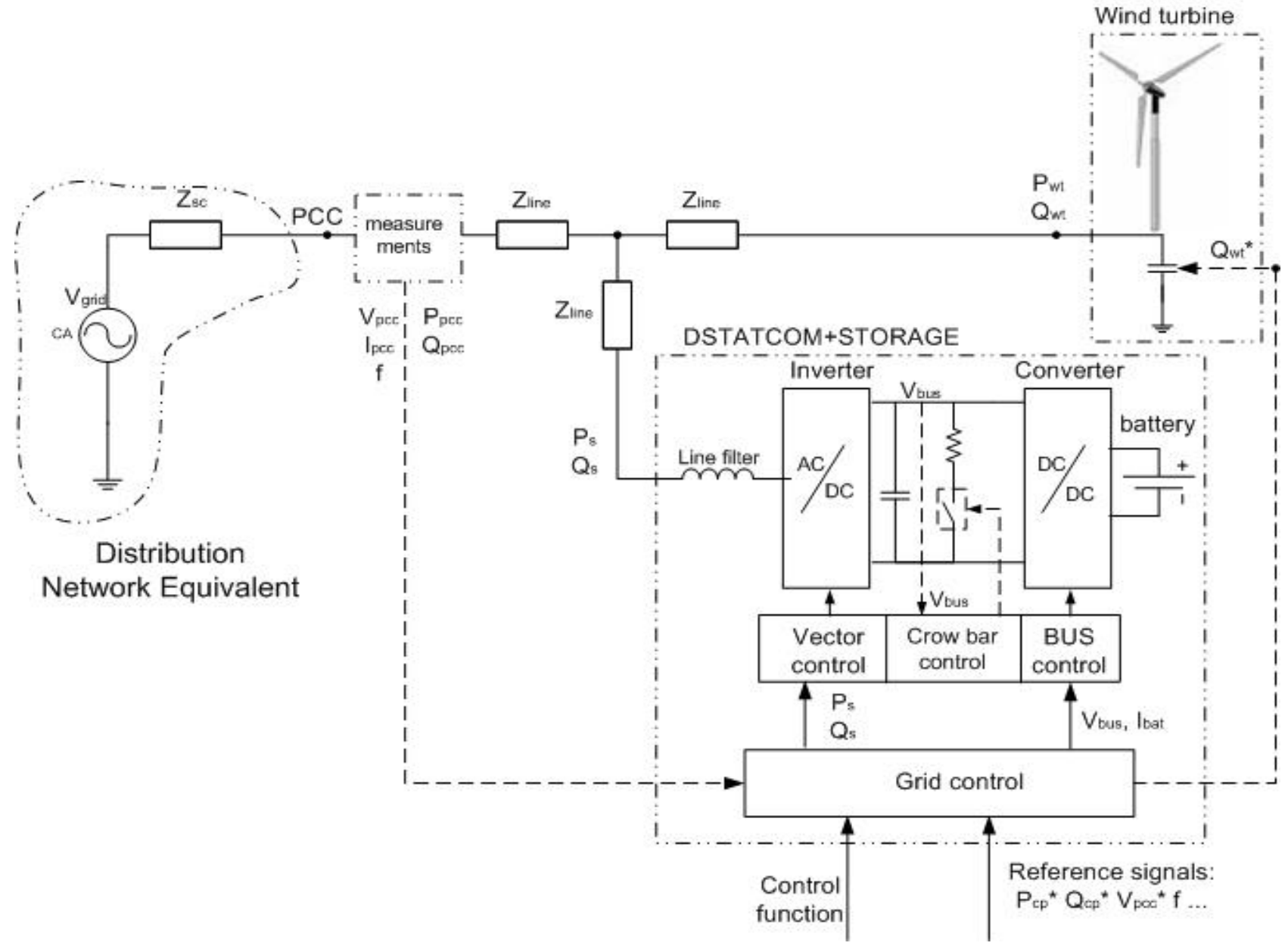

Figure 1. Topology of the system.

\section{System modelling}

In the present study electromagnetic transient simulation software will be used. MATLAB has been traditionally used for the simulation of control systems due to its facility of use and flexibility. In order to carry out power system analyses, it requires especial toolboxes (Power System Block-set) which are well adapted to the analysis of small power systems like the system of this study.

Next sections will describe the models of the different components of the system.

\section{A. Distribution grid}

The distribution network equivalent grid will be modelled in a first stage as an ideal voltage source with a short-circuit impedance with an adequate $\mathrm{X} / \mathrm{R}$ ratio. In this case the short circuit impedance is defined around the $10 \%$ of the nominal power of the wind turbine.

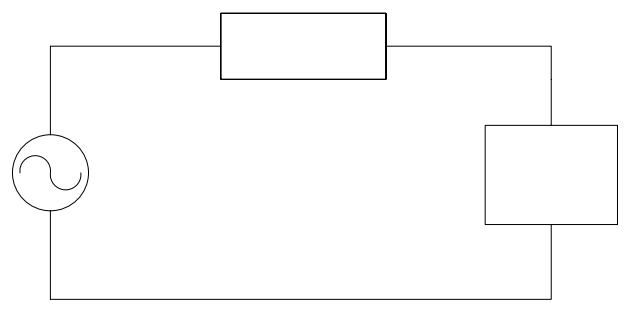

Figure 3: grid equivalent

$S_{\text {sc }}=10 \cdot S_{\text {nom }}$

$S_{s c}=3 \cdot \frac{V_{f f}^{2}}{Z_{s c}} \Rightarrow Z_{s c}=3 \cdot \frac{V_{f f}^{2}}{S_{s c}}$

The $\mathrm{X} / \mathrm{R}$ ratio is around 10 .

B. Wind turbine

The fixed speed turbine model is composed of five cascaded elements [6, 7]:

- Wind, aerodynamic system and mechanical system.

- Asynchronous generator. 
- Mechanically switched capacitor.

Mechanically switched capacitors (MSCs) represent in general a well-established solution to enhance the power factor correction for the wind turbine. In this case it will be controlled at grid control level.

The wind turbine analysed in this paper is a fixed speed $55 \mathrm{~kW}$ asynchronous generator, which works in a range of wind between 4 and $26 \mathrm{~m} / \mathrm{s}$ and it supplies its nominal power for around $12.5 \mathrm{~m} / \mathrm{s}$.

\section{DStatcom}

The DStatcom is composed of a three phases voltage source inverter connected to the grid by means of an inductive filter. In order to reduce the simulating time the modulator and the switches will be modelled as voltage controlled sources [7].

\section{Storage system}

The storage system is composed by the battery and the DC/DC converter. The converter is modelled by its medium model and the battery by means of an electrical model taking into account the battery lifetime and the voltage-current characteristics [8].

\section{Control specifications}

The main objective of the overall control system is to improve the interaction between the isolated grid (scenario) and the distribution grid. That means that it is necessary to deal with two types of situations: normal and fault operation described below. In this paper is analysed only the first case, the normal operation.

\section{E. Normal operation}

In this operation mode the exchange of active and reactive power in the point of common coupling (PCC) will be controlled. Several control functions are typically required for wind farms [9], and can be considered the initial control specification for our system considering the scenery proposed as a renewable generation unit.

The active power control functions required by the system operators are as follows:

- Balance control—whereby the PCC production can be adjusted downwards or upwards, in steps at constant levels.

- Delta control—whereby the complete system is ordered to operate with a certain constant reserve capacity in relation to its momentary possible power production capacity.
- Power gradient limiter-which sets how fast the complete system power production, can be adjusted upwards and downwards.

The reactive power control functions required by the system operators are:

- Reactive power control-the complete system is required to produce or absorb a constant specific amount of reactive power.

- Automatic voltage control-the voltage in the PCC is controlled. This implies that the complete system can be ordered to produce or absorb an amount of reactive power to the grid in order to compensate deviations on the grid voltage.

\section{Control strategies}

The control strategies are divided in two levels of control hierarchy:

\section{A. Converter control}

The converter control is responsible for active and reactive power exchange by the grid inverter control and the current control of battery.

Among all the control strategies proposed for the voltage source inverter, for this study a vector control has been selected [7]. In this case, it will receive as references, the active and reactive power supplied by the DStatcom (Ps and Qs).

The bus voltage will be controlled by means of the control of the battery current, so a bus voltage regulator will provide a battery current reference.

The crowbar control will act as a security system.

\section{B. Grid control}

The grid control has two main objectives:

- Adapt to our scenery and implement the grid functionalities described in the precedent section and manage the different operation modes.

- Manage the energy stored in the batteries.

It will also provide the references for Ps and Qs.

The main purpose of this paper is the development of the above mentioned control strategies.

Notice that for example the voltage control in distribution grids cannot be done by means of the reactive power due to the poor $\mathrm{X} / \mathrm{R}$ ratio and this presents an extra degree of difficulty [12].

\section{Simulations}

In order to analyse the above defined control specifications the system modelled in 
MATLAB/SIMULINK has been simulated for two different cases:

\section{A. Power control at the point of common coupling}

The objective of the control was to supply constantly $25 \mathrm{~kW}$ and $0 \mathrm{kVAr}$ to the point of common coupling (PCC). Since the STATCOM can supply $15 \mathrm{kVA}$ as much, to maintain the active power supplied to the PCC on $25 \mathrm{~kW}$, the power produced by the generator should be between $10 \mathrm{~kW}$ and $40 \mathrm{~kW}$. For this case was chosen a wind profile of more or less $7.5 \mathrm{~m} / \mathrm{s}$ with a perturbation that increases the wind speed up to $9.5 \mathrm{~m} / \mathrm{s}$ for an instant. With this wind profile (shown in figure 3) it is possible to see clearly how the power control works.

For the control of the power supplied by the STATCOM there have been implemented two loops to control the power. In the primary loops are compared the active and reactive power measured in the PCC with the power references, the errors of these comparisons are applied to two PI controllers, these controllers set the current references for the control of the STATCOM. In the secondary loop the voltage of the inverter is controlled by means of a vector control.

For the vector control is used a PLL to measure the angle of the voltage at the STATCOM in this way is possible to obtain the $\mathrm{d}-\mathrm{q}$ components of the current. These $\mathrm{d}-\mathrm{q}$ components are compared with the references coming from the primary control, and then these errors excite the PI controllers. These controllers set the $d-q$ components of the voltage defining the voltage that the inverter should supply.

In the following figures are shown the variations on the voltage module, on the active power and the reactive power, for different points of the micro grid: the wind turbine, the point of common coupling and the STATCOM.

As figure 3 shows the variation on the power produces a small variation in the power injected on the point of common coupling. During this perturbation the STATCOM absorbs the extra power that the wind turbine is producing, shaving the production peak of wind turbine. This simulation demonstrates how using a STACOM with energy storage is possible to control the power production of a renewable generation unit. In this way within the limits that renewable sources permit, it is possible to connect to the grid a stable power production.
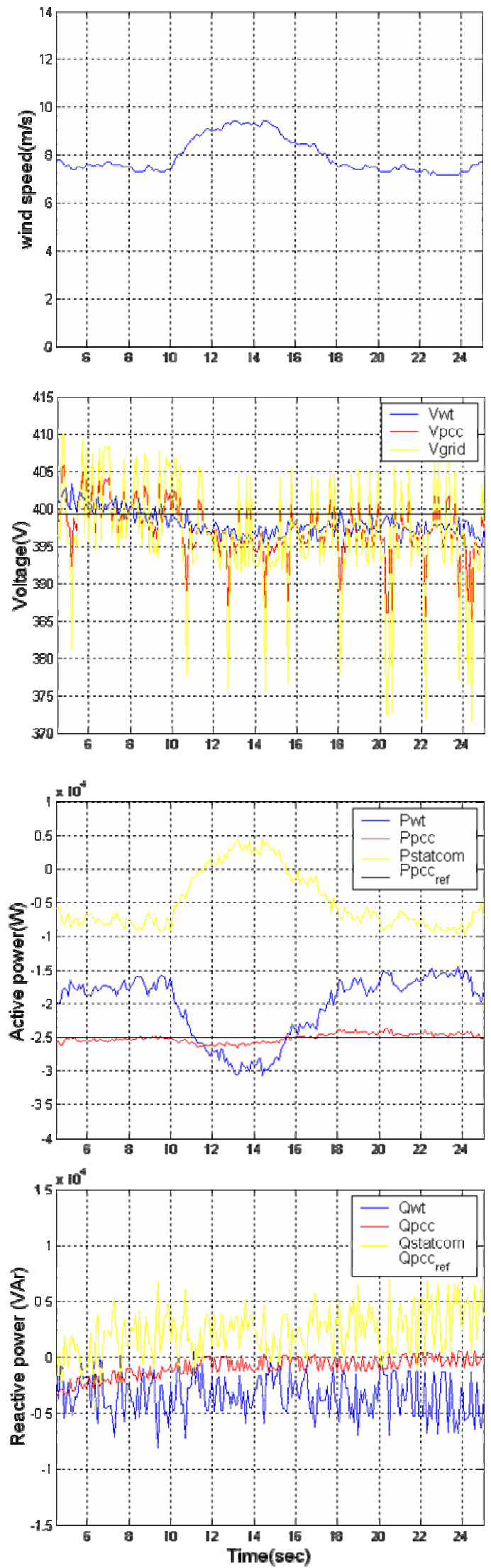

Figure 3: simulation of the power control at the point of common coupling 


\section{B. Voltage control at the point of common coupling}

The objective of the control was to maintain the voltage on $400 \mathrm{v}$ at the point of common coupling. For this case was chosen a wind profile of more or less $7.5 \mathrm{~m} / \mathrm{s}$ in the beginning, changing then to $15 \mathrm{~m} / \mathrm{s}$. With this wind profile (shown in figure 5) it is possible to check how the voltage control works for two different levels of power production. For this simulation the losses in the lines have been increased in order to obtain a larger voltage variation.

In figure 4 is shown how would change the voltage applying this wind profile to the wind turbine when there is not any supporting device.

For this simulation it has been used the same control as for the previous simulation. But in this case a reactive power voltage drop control supplies the reactive power reference for the point of common coupling. The active power reference is defined on zero, making the STATCOM work just as a voltage controller.

Depending on the difference between the voltage reference and the voltage measured on point of coupling it is set the reactive power reference, always within the limits of the STATCOM.

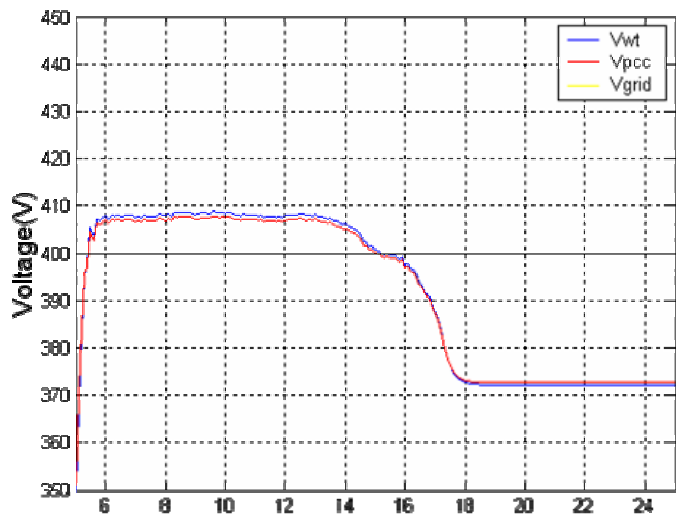

Figure 4: simulation of the voltage without any control

In the following figures are shown the variations on the voltage module, on the active power and the reactive power, for different points of the micro grid: the wind turbine, the point of common coupling and the STATCOM.

As figure 4 shows in a scenario of a weak grid connection point the STATCOM can control voltage making it be within acceptable limits.
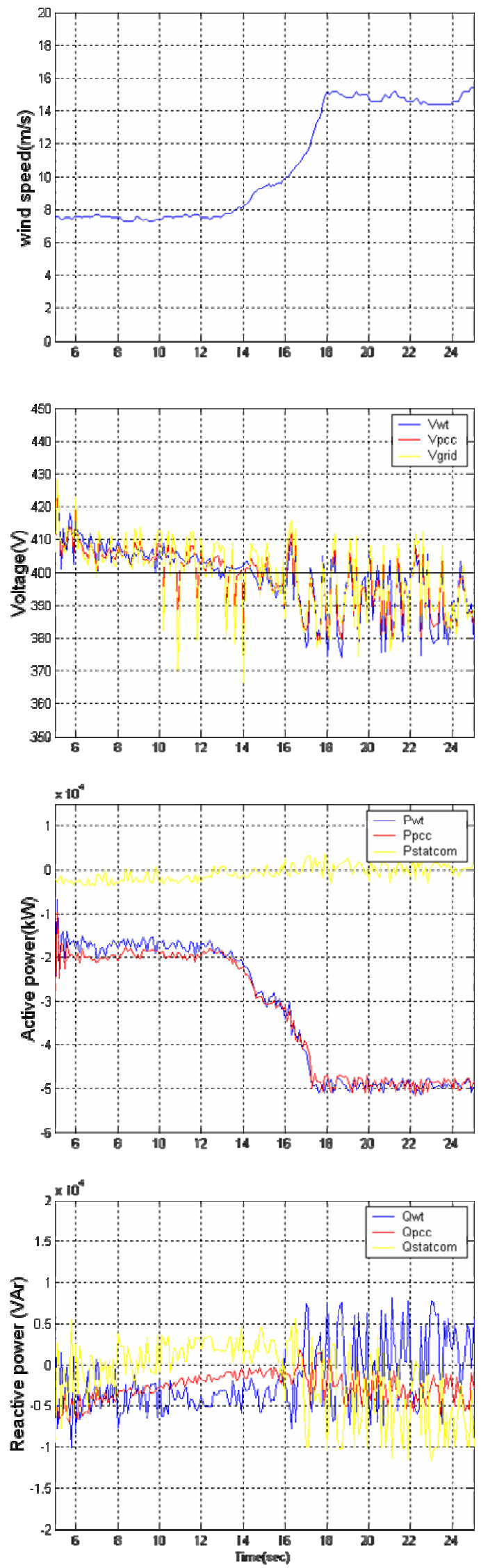

Figure 5: simulation of the voltage control at the point of common coupling 


\section{Conclusions and further works}

In conclusion a STATCOM with a storage system can help in the grid integration of renewable distributed generators, as it has been shown in this paper for the case of a $55 \mathrm{~kW}$ fixed speed wind turbine. With the correct sizing and placement of the supporting devices is possible to make renewable power generators work as intelligent power generators within the limits of its renewable resource.

Next steps will be:

- Develop a frequency control strategy.

- Analyse the fault behaviour.

In a further work a real time implementation in the Syslab facility will be performed.

\section{References}

[1] T. Ackerman, G. Andersson, L. Söder. "Distribution Generation: a definition". Electric Power System Research 57 (2001) 195-204. 2001.

[2] Arulampalam, A.; Barnes, M.; Jenkins, N.; Ekanayake, J.B.; "Power quality and stability improvement of a wind farm using STATCOM supported with hybrid battery energy storage”. Generation, Transmission and Distribution, IEE Proceedings- Volume 153, Issue 6, November 2006 Page(s):701 - 710.

[3] Oliver Gehrke, Philippe Venne and Stephanie Ropenus "Centralized and Distributed Control: A Power System Point of View”. Risø International Energy Conference, May 2007, Roskilde, Danmark.

[4] Henrik Bindner, Oliver Gehrke and Philippe Venne "Piecing it together: Communication for the distributed control of power systems" 2nd International Conference on Integration of Renewable and Distributed Energy Resources 4th - 8th December 2006 Napa, California, USA.

[5] Henrik Bindner "Vanadium Flow Batteries- Initial Results from Characterisation Measurements”. Risø International Energy Conference, May 2007, Roskilde, Danmark.

[6] J. G. Slootweg. Wind power modelling and generator power system dynamics; PhD Thesis 2003, Technical University of Delft.

[7] V. Akhmatov. "Analysis of dynamic behaviour of electric power systems with large amount of wind power”; PhD Thesis 2003, Electric Power Engineering, Ørsted-DTU, Technical University of Denmark.
[8] Min Chen, Gabriel a. Rincon-Mora. “Accurate electrical battery model capable of predicting runtime and $\mathrm{i}-\mathrm{v}$ performance”. Transactions on energy conversion, vol. 21, no. 2, June 2006

[9] Anca D. Hansen, Poul Sørensen, Florin Iov, Frede Blaabjerg. "Centralised power control of wind farm with doubly fed induction generators". Renewable Energy 31 (2006) 935-951.

[10] Florin Iov, Anca Daniela Hansen, Poul Sørensen, Nicolaos Antonio Cutululis."Mapping of grid faults and grid codes”. Risø-R-1617(EN)

[11] Gaztanaga, H.; Etxeberria-Otadui, I.; Ocnasu, D.; Bacha, S. "Real-Time Analysis of the Transient Response Improvement of Fixed-Speed Wind Farms by Using a Reduced-Scale STATCOM Prototype”. Power Systems, IEEE Transactions on. Volume 22, Issue 2, May 2007 Page(s):658 - 666.

[12] Fabio Bignucolo, Roberto Caldon, Valter Prandoni. "Radial MV networks voltage regulation with distribution management system coordinated controller”. Electric Power Systems Research xxx (2007) $\mathrm{xxx}-\mathrm{xxx}$ 
